\title{
Recent Trends towards Green Clouds by using Fuzzy based Live Migration
}

\author{
Amrinder Kaur \\ Department Of Computer Science \\ Guru Nanak Dev University, Amritsar (Pb.) 143005
}

\author{
Anil Kumar \\ Department Of Computer Science \\ Guru Nanak Dev University, Amritsar (Pb.) 143005
}

\begin{abstract}
Cloud computing is just a new field in Web computing that provides novel views in internetworking systems and improves problems in the architecture, style, and implementation of present communities and knowledge centers. It is the Internet based computing where essential shared servers provide software, infrastructure, platform, devices and other resources and offering to customers on a pay-as-you-use basis. It is often said that the cloud computing is a type of computing where scalable, flexible, and supple IT abilities are provided as a service to multiple customers. This paper presents a review on cloud computing. The overall objective of this work is to evaluate the gaps in earlier work in cloud computing and finding the suitable solution for the same.
\end{abstract}

\section{Keywords}

Cloud Computing, Energy Efficiency

\section{INTRODUCTION}

Cloud computing is providing utility-oriented IT companies to users worldwide. Centered on a pay-as-you-go model, it enables hosting of pervasive purposes from client, medical, and organization domains. Nevertheless, knowledge stores hosting Cloud purposes eat up large levels of power, contributing to large operational prices and carbon footprints to the environment. Thus, need Green Cloud computing solutions that could not only save power for the surroundings but additionally reduce operational costs. Concentrate on the development of vibrant source provisioning and allocation formulas that think about the synergy between different knowledge middle infrastructures (i.e., the hardware, power items, chilling and software), and holistically work to boost knowledge middle power efficiency and performance.

Cloud computing is just a new field in Web computing that provides novel views in internetworking systems and improves problems in the architecture, style, and implementation of present communities and knowledge centers. The appropriate research has just recently acquired momentum and the space of possible some ideas and solutions remains definitely not being commonly explored. Cloud computing is one of many fieriest, developing technology in the IT world. The term cloud is similar to the Internet. In genuine cloud computing model, this implies having all the software and knowledge used on a server or several servers, and accessing them through the internet. Sometimes the customer is merely a computer device furnished with a nominal OS and operating a web browser.

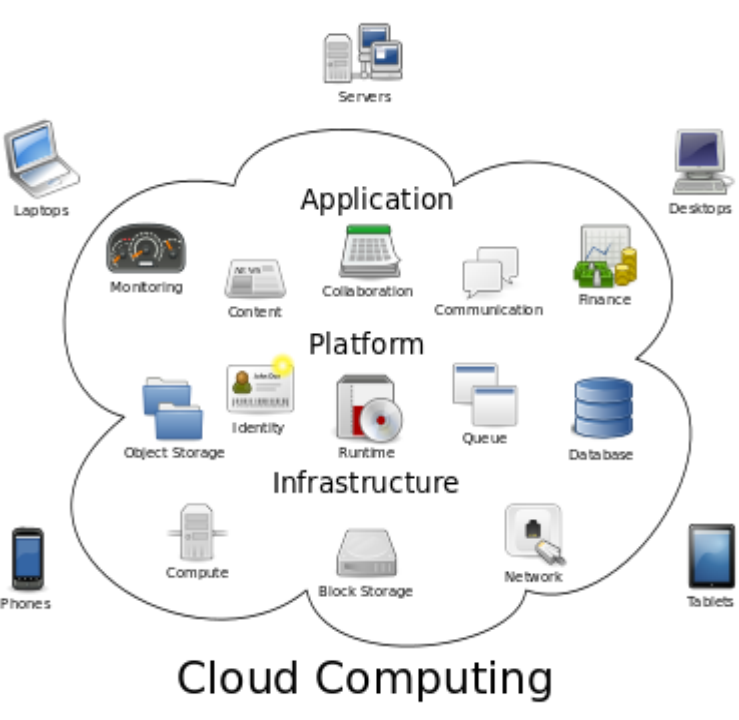

Figure1: Cloud Computing

Consumers can use these services on the cloud without understanding - how a handling of the methods is done. Ergo the customers can only emphasis on their control rather than wasting time and attaining information on the methods needed to cope up their processes. To see cloud computing is to consider the email account. To gain access to the consideration, open the visitor, visit the email customer and simply log-in and the important portion is to really have the Net access. The e-mail consideration isn't located i.e. the complete of the Aol or Gmail computer software isn't mounted on the pc as an alternative it is accessed by the Net connection.

\section{CLOUD COMPUTING CHARACTERISTICS}

\subsection{On-Demand}

A fundamental notion of the cloud computing is to provide the methods whenever there is need. From the user's point of view the accessible computing methods are almost endless i.e., the consumer doesn't worry about the pair of machines based at one site therefore it is the duty of the cloud computing provider to possess satisfactory methods to fulfil the needs of most their customers. Employing computing methods on-demand is one of the most preferred talents for a sizable number of enterprises since it removes the necessity for planning forward, obtaining, and repairing the methods they could require sooner or later in the future. This permits the consumer to avoid creating a useless investment in servers. 


\subsection{Pay-per-use}

In the event of cloud computing the consumer gives only on the foundation of the usage while in the case of the traditional computing there is a need certainly to identify the methods literally on the consumer side. The cloud computing supplies the ability of paying the supplier relying only on the usage of the customer.

\subsection{Rapid Strength}

The cloud provider machines up or down the methods depending upon the precise of a site stage contract which can be offered for the adjusting customer needs. That support becomes the full time for the cloud provider to result back. Such an agreement is required by the cloud provider, as the cloud provider doesn't in reality have numerous methods, so depending upon the support stage contract the provider has to discover a couple of distributions of methods that fulfill the demands of the customers usually the support stage contract need a penalty that the provider has to cover to each customer for maybe not conference the agreement.

\subsection{Preservation and upgrading}

The provider keeps the computing resource rather than the user. Ergo the provider keeps and revisions the resources. Ergo all development of the methods are hidden from the user's see, but this may be true in the ideal case. Often due to a number of the causes the consumer is shifted in one system to the other, in that case the consumer might be supplied with any resource.

\section{CLOUD COMPUTING SERVICES}

Every company gives a certain purpose enabling individual to own less or even more control on the cloud with respect to the form i.e. individual, community, hybrid or community. The cloud need vary depending on what the cloud will be used i.e. the area and the methods related with the cloud. Cloud research could be classified by the model of company it provides into numerous groups. They're defined utilizing the XaaS taxonomy, wherever " $X$ " could be Computer software, Software, or Infrastructure, and the final "S" is for Service.

Along with above some professionals list these blocks of cloud research:
1. Storage-as-a-Service
2. Database-as-a-Service
3. Information-as-a-Service
4. Process-as-a-Service
5. Application-as-a-Service
6. Integration-as-a-Service
7. Security-as-a-Service
8. Management/Governance-as-a-Service
9. Testing-as-a-Service

\subsection{Computer Software as a Service (SaaS)}

A SaaS provider gives subscribed or pay-as-you-use individual use of both methods and services. SaaS causes it to be preventable to truly have a physical copy of pc software to set up on the devices. SaaS makes simpler to have the same pc software on all of your devices at once by opening it on the cloud. In a SaaS contract, one have the least control within the cloud. A typical example of SaaS is Bing Docs. It is just a party that's free for anyone to use. Making reports is free. By login-in use of the term model, spreadsheet is given. These numerous on line companies given by google are accomplished from the slim customer i.e. internet browser and involves no installation.

\subsection{Software as a Service (Paas)}

A PaaS program is above the Computer software as a Service arrangement. A PaaS provider gives members entry to the components that they might require to grow and purpose applications within the internet. One of many frequent exemplary instance of $\mathrm{PaaS}$ is Facebook. Makers and developers may create any software for the Facebook program applying APIs and make that application available to the users.

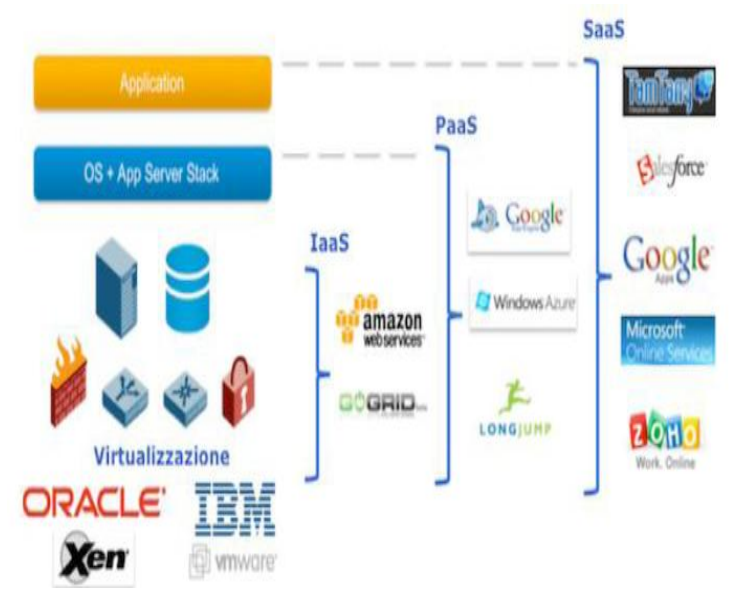

Figure 2: Cloud Computing service Model

\subsection{Infrastructure as a Service}

An IaaS system offers mostly with computational infrastructure. It supplies the client to fully outsource the storage and resources that they desire for computation. An example of IaaS is Amazon EC2. From the tiny to full-blown web sites, it offered the cloud infrastructure ability to perform them all.

\subsection{Computer software as a Service}

Computer software as a Service (SaaS) is the design in which a credit card application is used as a service that will be offered to customers who can have use of it via the Internet. When the application is presented off website, the customers do not need to uphold it or care for it. On another hand, it is not in the customer's hands once the hosting service chooses to improve it. The idea is to use the application because it is not essential to produce plenty of improvements or require incorporation to other system. The service does all the fixing and developments as well as keeping the infrastructure running.

The price for utilizing the application is the on-going method i.e. the user purchase the service just as much it has been used. Instead of paying it when, it is like the more the service has been applied, the more it be billed. You can find various kinds of application offering themselves to the SaaS model. Consumers who are not liable to perform application development but need purposes, they use SaaS. The main advantageous asset of SaaS is nothing apart from costing less money as opposed to purchasing the entire application. Some of the advantages of SaaS are: 


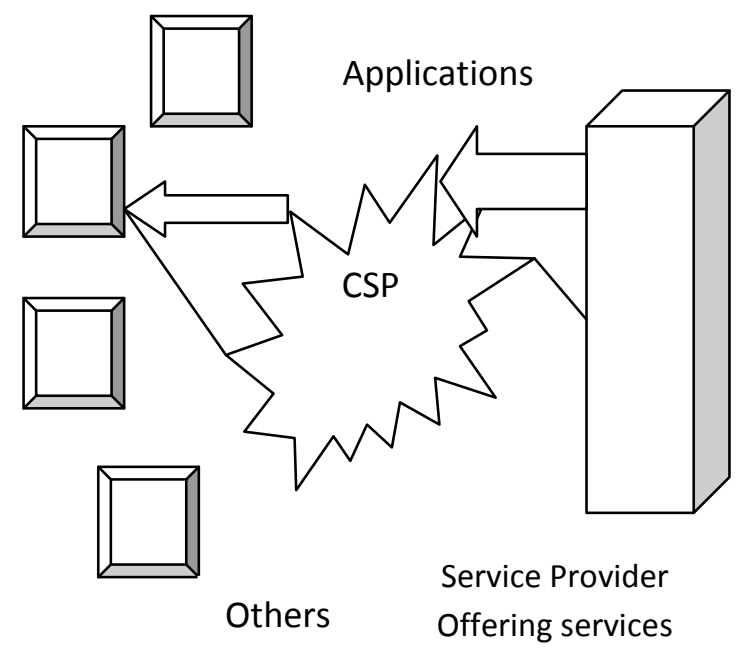

Figure 3: SaaS service provider

A. Familiarity with the World Wide Internet: Everyone have use of the computer and knows how to use Internet. Therefore supporting the clients to use the services. Smaller Team: IT techniques typically involve small staff because of the expense associated with the salaries and different benefits. The capability to variety the programs reduces the requirement of such large IT staff.

B. Modification: SaaS programs are typically customizable as set alongside the older ones. Greater Advertising: With the SaaS the programs are open to all or any the clients that has been early in the day difficult as the creator who has marketed the tiny application may have had an issue advertising the large application.

C. Protection: The Secure socket layer (SSL) is used. This enables the clients to achieve their programs firmly without having to hire complicated back end configuration. More Bandwidth: As the bandwidth is increasing, this permits the people to get into the programs with reduced latencies and excellent speeds.

D. Problems of SaaS: Like everything, SaaS also possess some problems in their employment and use. It could be possible that the organization that includes a unique need may not available through SaaS. In that situation they may need to get the application in general and deploy on the given machine. It are often possible that the customer have taken care of the applying but later they are incapable of dock that application to the brand new vendor.

\section{DEPLOYMENT MODELS}

Clouds may also be labeled based on the underlying infrastructure implementation product as Community, Individual, Neighborhood, or Cross clouds. The different infrastructure implementation versions are distinguishing by their structure, the located area of the datacenter where the cloud is noticed, and the wants of the cloud provider's clients (for case, due to regulatory, legal, and other requirements).

\subsection{Community Clouds}

A public cloud's physical infrastructure is possessed by a cloud service provider. Such a cloud goes programs from various clients who share this infrastructure and pay for their reference utilization on a utility computing basis.

\subsection{Individual Clouds}

A pure personal cloud is made for the exceptional usage of one customer, who owns and fully controls this cloud. Furthermore, there are modifications with this in terms of ownership, function, etc. The truth that the cloud is used by a certain customer could be the distinguishing feature of any personal cloud. An exclusive cloud may be possessed by the customer, but created, fitted, and handled by a third party rather than the customer. The physical hosts may be found at the customer's premises or sited in a collocation facility recently presented alternative to a private cloud is a 'electronic personal cloud '. In such a electronic personal cloud a person is allotted a private cloud within the physical infrastructure of a public cloud. Because of the allocation of unique resources within the cloud the customer may be sure that their information stored on and processing is done only on committed hosts (i.e., these hosts are not distributed to some other customer of the cloud provider).

\subsection{Neighborhood Clouds}

When several clients have similar requirements, they are able to share an infrastructure and might share the setting and management of the cloud. That management might be done independently or by third parties.

\subsection{Hybrid Clouds}

Finally, any composition of clouds, be they private or public, could form a hybrid cloud and be managed a single entity, provided that there is sufficient commonality between the standards used by the constituent clouds.

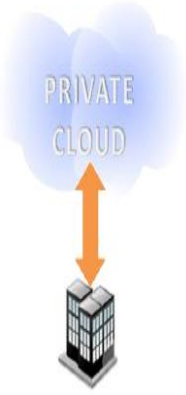

Private Cloud

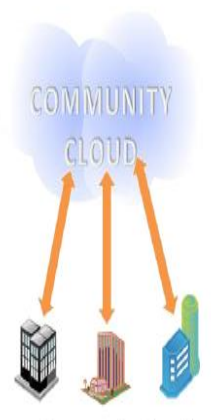

Community Cloud

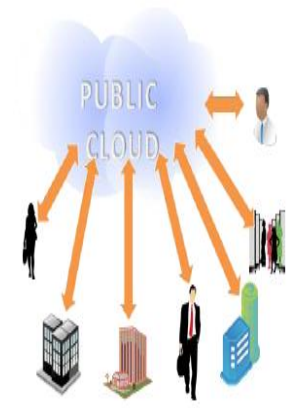

Public Cloud
Figure4 - Deployment models

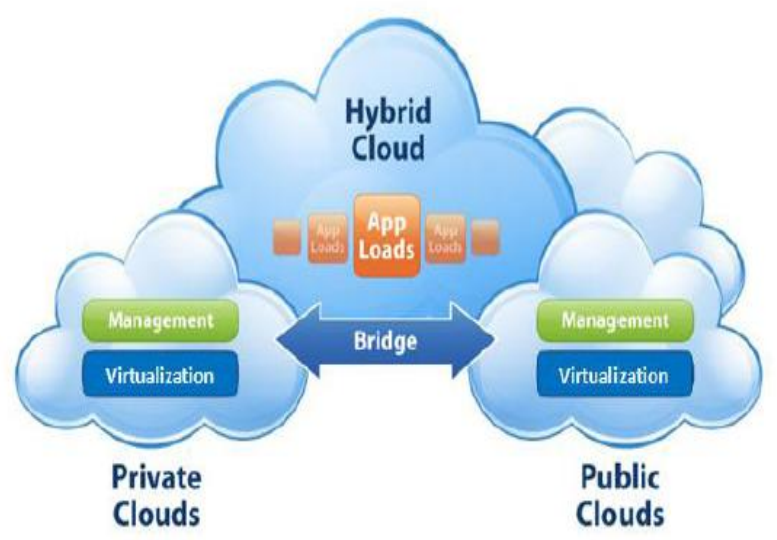

Figure5 - Hybrid clouds 


\section{PROPOSED WORK}

Cloud computing is currently emerging as a powerful way to transform the IT industry to build and deploy custom applications. The word cloud is similar to the Internet. But in real cloud computing model, it means to have all the software and data held on a server or a group of servers, and to access them through the internet. In certain cases the client is a device furnished with a nominal OS and running a web browser. Most of the distinct scheduling algorithm for efficient data center resources has used bivalent logic. The use of live migration in energy efficient scheduling is also based on bivalent theory. The use of fuzzy theory has also neglected in existing research of dynamic load balancing in cloud computing. This dissertation has focused on fuzzy based energy- Efficient scheduling algorithm with the live migration to balance the user queries in an optimistic manner. As it is known in prior that the fuzzy is based on multi-valent logic, so the proposed algorithm can easily overcome the limitation of existing techniques. Fuzzy logic will produced a fuzzy membership value. Every time to balance the load in efficient manner. Also, fuzzy logic will improve the live migration by developing fuzzy based decision making whom to shift the load. Due to non-availability of actual Cloud Environment, Simulation environment will be design and implement in MatLab tool with the help of data analysis toolbox.

\section{RELATED WORK}

R. Buyya et al. [1] presented vision, challenges, and architectural components for energy-efficient administration of Cloud computing environments. They focused on the growth of powerful reference provisioning and allocation formulas that considered the synergy between different information center infrastructures and holistically perform to enhance information center energy performance and performance. Specifically, they planned architectural maxims for energy-efficient administration of Clouds; energyefficient reference allocation procedures and arrangement formulas considering quality-of-service expectations, and products energy application characteristics; and a novel software technology for energy-efficient administration of Clouds. They have validated approach by completing a couple of arduous performance evaluation examine utilizing the Cloud-Sim toolkit. The outcome shown that Cloud computing model had immense potential because it offers substantial performance gets as regards to response time and charge preserving below powerful workload scenarios.J. Baliga et al. [2] presented an analysis of energy consumption in cloud computing. The analysis considered both public and personal clouds, and contains energy consumption in switching and signs along with information running and information storage. They revealed that energy consumption in transportation and switching can be a substantial percentage of complete energy consumption in cloud computing. Cloud computing could enable more energyefficient use of computing energy, particularly when the computing projects are of reduced intensity or infrequent. However, below some circumstances cloud computing could eat more energy than mainstream computing wherever each person performed all computing on their possess pc (PC).I.S. Moreno et al. [3] presented an energetic reference provisioning system to over allocate the capability of realtime Cloud information centers predicated on client operation patterns. Additionally, their affect the trade-off between energy performance and SLA fulfillment is analyzed. The key strategy is always to exploit the reference operation habits of every client to decrease the spend made by reference demand overestimations. This creates the opportunity to spend extra VMs in the exact same host incrementing their energy efficiency. Nonetheless, and also this increases the chance of QoS affectations. The planned model considers SLA deadlines, forecasts predicated on historical information, and powerful occupation to ascertain the total amount of methods to over allocate for every host. In addition, a compensation system to regulate reference allocation in instances of underestimation can be described. In order to consider the model, simulation analysis was conducted. Effects show important improvements in energyefficiency while SLA-deadlines are slightly impacted. However, in addition they place the importance of best compensation procedures to reduce availability violations particularly during top operation periods. I.Sarji et al. [4] planned two energy models predicated on a statistical analysis of a server's functional conduct in order to reduce the vitality consumption in information centers at cloud computing providers. Predicated on these models, the Power Savings Motor (ESE) in the cloud company decides either to migrate the electronic devices (VMs) from a lightly-loaded host and then transform it down or use it in a sleep method, or to help keep the present host running and ready to get any new load requests. The key big difference between the two models is the vitality and time needed to place the host in functional method from a sleep method or from an down state. Thus, the decision is just a tradeoff between the vitality savings and the mandatory performance in line with the SLA between the client and the cloud provider. They showed results predicated on genuine energy proportions taken at the server's AC insight, to ascertain the vitality eaten in the lazy state, the rest state, the down state and in case of switching between any two of the states. In addition, they tested the power eaten by the foundation and the location servers during the migration of a VM.F. Owusu et al. [5] discussed one section of conflict; the vitality performance of cloud computing. They outlined past contributions to the conversation of energy performance of cloud computing, give a functioning explanation of cloud computing and discuss their value, which will grow whilst the technology matures and becomes properly knownK. L. Keville et al. [6] analyzed the utilization of ARM-based clusters for lowpower, powerful computing. This work examines two probably use-modes: a regular devoted chaos, and a group of pre-configured electronic machines in the cloud. A 40-node department-level chaos based on an ARM Cortex-A9 is compared against a similar chaos based on an Intel Core2 Duo, in comparison to a current related examine on just a 4node cluster. For the NAS criteria on 32-node clusters, ARM was found to truly have a energy efficiency which range from 1.3 to 6.2 occasions greater than that of Intel. That is despite Intel's approximately five occasion's higher performance. This efficiency ratio depends generally on how big is the working collection in accordance with L2 cache. Along with energy-efficient research, that examines also highlights fault tolerance: a significant element in powerful computing. It utilizes two new extensions to the DMTCP checkpoint-restart package. DMTCP was extensive to aid ARM CPUs, and to aid check going of the Qemu electronic device in user-mode. DMTCP can be used both to checkpoint native spread purposes, and to checkpoint a system of electronic machines. This latter case shows the capability to use pre-configured pc software in electronic machines managed in the cloud, and more to move chaos computation between hosts in the cloud. W. Xiaoli et al. [7] recognized the power consumption design in cloud research atmosphere, and examined the power use in model. On the basis of the analysis, they improved the 
Online Bin Loading algorithm, and planned a new energyaware strategy with concern of energy efficiency in cloud research atmosphere which consists of electronic machines. At the conclusion, they demonstrated place strategy by simulation results. The simulation effects show this algorithm can not merely increase resource usage charges, but additionally produce the information middle more energy-efficient. S. R. Tucker et al. [8] mentioned about the power consumption and efficiency in interaction networks; trends, challenges and possibilities shown by the evolution to energy-efficient telecommunications; and cloud computing. T. Knaught et al. [9] used simulation to assess the huge difference in energy consumption triggered entirely by electronic device schedulers. Besides demonstrating the inadequacy of wide-spread standard schedulers, they shown optimized scheduler. Using a range of reasonable simulation cases, their tailored scheduler OptSched paid off cumulative device uptime by as much as $60.1 \%$. They evaluated the effect of data middle composition, run time circulation, electronic device sizes, and portion requests on cumulative device uptime. IaaS administrators can use their brings about rapidly assess probable savings in device uptime and, ergo, untapped energy saving potential. A.K.Das et al. [10] created an flexible QoS (Quality of Service) conscious VM provisioning system that ensured efficient usage of the device resources. The VM for related form of requests have been recycled so your VM creation time could be minimized and used to serve more consumer requests. In the planned design, QoS was ensured by offering all of the jobs within the requirements defined in SLA. Responsibilities were divided using multilevel line and the absolute most urgent job was presented with large priority. The simulation-based experimental effects showed a great number of jobs could be served compared to the others which would help satisfy consumers through the peak hour. T.Adhikari et al. [11] have planned a system for chaos formation based on system location among the information servers. They created two spread and local intra-cluster and inter chaos VM scheduling calculations based on energy formula, resource requirement and availability. Their planned scheduling calculations control VMs to reduce the power consumption of the hosts and network devices. Simulation effects revealed that the planned spread VM scheduling calculations conserved significant level of energy compared to state-of-the artwork worksK. Negin et al. [12] proposed an energy-efficient approach predicated on Minimum Connection Coefficient (MCC) process for virtual machine position in cloud centered, virtualized data centers. The proposed approach regards equally Service Level Deal (SLA) and minimal energy usage and attempts to produce a trade-off between those two problems applying fuzzy Analytic Hierarchy Method (AHP). They examined approach applying Cloudsim toolkit as a contemporary cloud processing environment simulator. The evaluation suggests that their proposed process offers an acceptable trade-off between energy performance and SLA violation decrease in cloud data centers. T. Arthi et al. [13] analyzed the prevailing energy usage model for various forms of companies in cloud processing environment and they shown a new energy-aware provisioning approach by considering energy performance as a vital factor. Their model uses pre-processed data about the support use of cloud useful for initiating the live migration. Their style encompasses the aspect named as induce engine which initiates intelligent migration of $\mathrm{VM}$ to maintain the processing environment natural and energy friendly. M. Barbulescu et al. [14] used the chance of applying Cloud Computing and

Grid Computing for calculations of finesse in energy performance. The report investigated a number of the principles of cloud processing with purpose of introducing cloud processing in spread system. M. Kaur et al. [15] proposed a new model for energy performance of cloud processing that keeps track how much amount of deterioration of atmosphere has occurred by the emissions of numerous natural home gases by various huge data centers and how processing may become eco- friendly. That report actually discovers the various essential issues that develop when such energy-saving methods are lengthy for use in cloud processing techniques. W. Keho et al. [16] developed an energy usage type of the Cloud Computing process, by using mathematical process may calculate the energy use of a digital machine in a tiny array of errors in 3\%-6\%. Then, on the basis of the model, they proposed a digital machine scheduling algorithm to improve the energy performance of the system. First, they collection a ceiling price of energy usage for every single machine in the system, and by analyzing these perform ideas published by each virtual machine, they tested if the ceiling can been exceeded or not. Then, by migrate one/several selected virtual products to other bodily servers in the system they can reduce steadily the energy use of the entire system. Their evaluation suggests that the proposed scheduling algorithm may efficiently implement energy-saving objectives without substantial decrease of the Quality of Services.

\section{CONCLUSION AND FUTURE SCOPE}

With cloud computing, an organization has the capacity to install computers for the employees and instead of installing applications or application on the individual computers, a single request has the capacity to be loaded on multiple computers. That not only diminishes the software and equipment needs of the user's device but also develops the major training of application in to most of the other computers in the system therefore making the user's device a lot more efficient. That workload change has observed many corporations resorting in to cloud computing. The paper presents the survey on various cloud computing issues. This survey has shown that the most of the existing researchers has neglected the scalability of cloud users and energy efficiency in cloud computing environment. The use of live migration in energy efficient scheduling is also based on bivalent theory. So in near future we will extend this work to overcome this constraint of bivalent by using fuzzy values. The use of fuzzy logic seems to be efficient as it will come up with best alternative for shifting the load from one position to another. Also the use of fuzzy logic will reduce the overall overheads of the live migration techniques.

\section{REFERENCES}

[1] Buyya, Rajkumar, Anton Beloglazov, and Jemal Abawajy. "Energy-efficient management of data center resources for cloud computing: A vision, architectural elements, and open challenges." arXiv preprint arXiv:1006.0308 (2010).

[2] Baliga, Jayant, Robert WA Ayre, Kerry Hinton, and RodneyS Tucker. "Green cloud computing: Balancing energy in processing, storage, and transport." Proceedings of the IEEE 99, no. 1 (2011): 149-167.

[3] Moreno, Ismael Solis, and Jie Xu. "Customer-aware resource over allocation to improve energy efficiency in 
real-time cloud computing data centers." In ServiceOriented Computing and Applications (SOCA), 2011 IEEE International Conference on, pp. 1-8. IEEE, 2011.

[4] Sarji, I., C. Ghali, A. Chehab, and A. Kayssi. "CloudESE: Energy efficiency model for cloud computing environments." In Energy Aware Computing (ICEAC), 2011 International Conference on, pp. 1-6. IEEE, 2011.

[5] Owusu, Francis, and Colin Pattinson. "The current state of understanding of the energy efficiency of cloud computing." In Trust, Security and Privacy in Computing and Communications (TrustCom), 2012 IEEE 11th International Conference on, pp. 1948-1953. IEEE, 2012.

[6] Keville, Kurt L., Rohan Garg, David J. Yates, Kapil Arya, and Gene Cooperman. "Towards Fault-Tolerant Energy-Efficient High Performance Computing in the Cloud." In Cluster Computing (CLUSTER), 2012 IEEE International Conference on, pp. 622-626. IEEE, 2012.

[7] Xiaoli, Wang, and Liu Zhanghui. "An energy-aware VMs placement algorithm in Cloud Computing environment." In Intelligent System Design and Engineering Application (ISDEA), 2012 Second International Conference on, pp. 627-630. IEEE, 2012.

[8] Tucker, Rodney S., Kerry Hinton, and Rob Ayre. "Energy efficiency in cloud computing and optical networking." In European Conference and Exhibition on Optical Communication, pp. Th-1. Optical Society of America, 2012.

[9] Knauth, Thomas, and Christof Fetzer. "Energy-aware scheduling for infrastructure clouds." In Cloud Computing Technology and Science (CloudCom), 2012 IEEE 4th International Conference on, pp. 58-65. IEEE, 2012.

[10] Das, Amit Kumar, Tamal Adhikary, Md Abdur Razzaque, and Choong Seon Hong. "An intelligent approach for virtual machine and QoS provisioning in cloud computing." In Information Networking (ICOIN), 2013 International Conference on, pp. 462-467. IEEE, 2013.

[11] Adhikary, Tamal, Amit Kumar Das, Md Razzaque, and A. M. Sarkar. "Energy-Efficient Scheduling Algorithms for Data Center Resources in Cloud Computing." In High Performance Computing and Communications \& 2013 IEEE International Conference on Embedded and Ubiquitous Computing (HPCC_EUC), 2013 IEEE 10th International Conference on, pp. 1715-1720. IEEE, 2013

[12] Kord, Negin, and Hassan Haghighi. "An energyefficient approach for virtual machine placement in cloud based data centers." In Information and Knowledge Technology (IKT), 2013 5th Conference on, pp. 44-49. IEEE, 2013.

[13] Arthi, T., and H. S. Hamead. "Energy aware cloud service provisioning approach for green computing environment." In Energy Efficient Technologies for Sustainability (ICEETS), 2013 International Conference on, pp. 139-144. IEEE, 2013.

[14] Barbulescu, Mihai, Ramona-Oana Grigoriu, Giorgian Neculoiu, Ionela Halcu, Virginia Cristiana Sandulescu, Oana Niculescu-Faida, Mariana Marinescu, and Viorel Marinescu. "Energy efficiency in cloud computing and distributed systems." In Networking in Education and Research, 2013 RoEduNet International Conference 12th Edition, pp. 1-5. IEEE, 2013.

[15] Kaur, Manjot, and Prabhdeep Singh. "Energy efficient Green Cloud: Underlying structure." In Energy Efficient Technologies for Sustainability (ICEETS), 2013 International Conference on, pp. 207-212. IEEE, 2013.

[16] Wu, Kehe, Ruo Du, Long Chen, and Su Yan. "An Energy-Saving Virtual-Machine Scheduling Algorithm of Cloud Computing System." In Information Science and Cloud Computing Companion (ISCC-C), 2013 International Conference on, pp. 219-224. IEEE, 2013. 